\title{
Korrelation der Thromboplastinzeiten bei Dicumarol-behandelten Patienten unter Verwendung verschiedener Thrombokinase-Präparate
}

\author{
Von R. Averdunk und K. Borner \\ Aus dem Institut für Klinische Chemie und Klinische Biochemie der Freien Universität Berlin, Klinileum Steglitz
}

(Direktor: Prof. Dr. H.-J. Dulce)

(Eingegangen am 20. Februar 1970)

1. 77 Proben von mit Dicumarin-Derivaten eingestellten Patienten wurden gleichzeitig mit 7 Thrombokinase-Präparaten im Quicktest untersucht. Die Ergebnisse wurden statistisch auf ihre Vergleichbarkeit (Korrelation und Regression) überprüft. Die mit den verschiedenen Thrombokinasen ermittelten Quickwerte korrelieren schr unterschiedlich miteinander. Der Korrelationskoeffizient $r$ schwankt zwischen 0,628 und 0,910. Es werden Tabellen und Umrechnungsfaktoren für die Thrombokinasc-Präparate der Firmen Behring, Geigy, Hoffmann-La Roche, Gödecke (Simplastin), Hyland und Nygărd (Thrombotest) angegeben.

2. Die Besonderheiten der Regressionsanalyse für den Fall, daß beide zu vergleichenden Variablen mit einer Unsicherheit gleicher Größenordnung behaftet sind, werden beschrieben.

\section{Correlation of the thromboplastin times in dicumarol-treated patients, using different thrombokinase preparations}

1. 77 Samples from patients treated with dicumarol derivates were studied all at the same time in the Quick test with 7 different thrombokinase preparations. The results were tested statistically for their comparability (correlation and regression). Quick values obtained with different thrombokinases showed a very variable correlation with each other. The correlation coefficient, $r$, varied between 0.628 and 0.910. Tables and conversion factors are given for the thrombokinase preparations from Behring, Geigy, Hoffmann-La Roche, Gödecke (simplastin), Hyland and Nygărd (thrombotest).

2. Special treatment is described for the case where the two variables to be compared arc both subject to the same order of uncertainty.

Die Behandlung mit Antikoagulantien vom CumarinTyp erfordert exakte Úberwachung der Dosierung durch Bestimmen der Thromboplastinzeiten (Quicktest). Es ist eine Anzahl von Thrombokinasepräparaten im Handel, die bei derselben Probe eines Patienten unterschiedliche Resultate liefern (1-4). Bei Patienten, die Wohnort oder Arzt wechseln, sollte man die mit einem Präparat gewonnenen Ergebnisse in die Skala eines zweiten Präparates umrechnen können. Als spezielles Problem kommt die Umrechnung von Thrombotest-Ergebnissen auf andere Analysen hinzu: wir sind z. B. aus organisatorischen Gründen gezwungen, zwei verschiedene "Quickteste" zu verwenden, den Thrombotest als Mikromethode für ambulante Patienten und einen MakroQuicktest bei stationären Patienten. Die vorliegende Arbeit versucht, die Ergebnisse von Quickwerten, mit verschiedenen Thrombokinasen bestimmt, zu korrelieren. Das Untersuchungsmatterial stammt von Cumarinbehandelten Pạtienten. Eine Bewertung der einzelnen Präparate im Sinne der Zuverlässigkeit ist nicht Ziel der Untersuchung. Auf die in diesem Zusammenhang-auftretenden Probleme der bivariaten Regressionsanalyse wird gesondert eingegangen.

\section{Material und Methoden}

\section{Untersuchungsmaterial und Patientengut}

Proben von 80 ambulanten Patienten, die mindestens 6 Wochen unter Cumarin-Therapie standen und deren Werte, gemessen mit der Thrombotest-Mikromethode, zwischen 5 und 20\% der Norm lagen, wurden untersucht.

\section{Bestimmung der Thromboplastinzeit}

Zur Blutentnahme wurde Citratblut (1 Teil 0,1м Natriumcitratlösung +9 Teile Blut) in einer Plastikspritze (Braun, Melsungen) entnommen. Nach Zentrifugieren des Blutes in silikonierten Glasgefäßen (10 Min. bei $1500 \mathrm{~g}$ ) wurde das überstehende Plasma in silikonierte Reagenzgläser dekantiert. Das Eintreten der Gerinnung wurde bei $37^{\circ}$ automatisch mit dem Koagulometer der Firma Schnittger, Göttingen, gemessen. Plasma und Thromboplastin wurden mit Marburg-Pipetten (Firma Eppendorf, Hamburg) eingefüllt. Die Bestimmung der Normprozente erfolgte über eine Eichkurve, die durch Verdünnen eines Gemisches von 10 Plasmen gesunder Probanden erstellt wurde. Es wurden Meßwerte mit 100, 80, 60, 50, 40, 30, 20 und $10 \%$ der ursprünglichen Plasmakonzentration erstellt. Die Eichkurve wurde auf doppelt-logarithmischem Papier gezeichnet. Für den Thrombotest wurden die vom Hersteller mitgelieferten Eichkurven zugrunde gelegt. Zum Vergleich wurden die Thrombokinasen folgender Firmen verwendet ${ }^{1}$ ):

1. Thrombotest Nyko der Firma Nygărd, Oslo, bezogen über die Firma Dr. Molter GmbH., Heidelberg

2. Simplastin der Firma Gödecke Charge Nr. 0432068

3. Thromboplastin Dade. Asid-Institut GmbH., München Charge $133 \mathrm{~A}$ und Charge AH 2-140-16-AB

4. Thromboplastin Hyland. Travenol International $\mathrm{GmbH}$. Charge 0357 Do 42 A1 und 0357 E 006 A1

5. Thromboplastin-Lösung La Roche. Hoffmann-La Roche, Basel Charge Nr. 6088

6. Thrombokinase Geigy. Geigy AG., Basel Charge Nr. $180801 \mathrm{Bk}$

7. Calcium-Thromboplastin Behring-Werke AG., Marburg/Lahn Charge Nr. 307 A

1) Für die genannten Präparate werden nachfolgend die Abkürzungen benutzt: 1 . Thrombotest, 2. Simplastin, 3. Dade, 4. Hyland, 5. La Roche, 6. Geigy, 7. Behring. 


\section{Statistisclie Methoden}

Von allen Analysengruppen wurden Mittelwert ( $\overline{\mathrm{x}})$, Standardabweichung (s) und Variationskoeffizient (VK) nach Gleichung (1), (2) und (3) berechnet. Hierbei wurden alle Ergebnisse verwendet.

$$
\begin{aligned}
\bar{x} & =\frac{1}{n}\left[x_{1}\right] \\
s & =\sqrt{\frac{\left[x_{1}-\bar{x}\right]^{2}}{n-1}} \\
V K & =\frac{100 \cdot s}{\bar{x}}
\end{aligned}
$$

Für $\overline{\mathrm{X}}$ werden nachfolgend sinngemäß auch $\overline{\mathrm{f}}$ und $\overline{\mathrm{q}}$ verwendeț. Zur Regressionsanalyse wurden die $\left(\begin{array}{l}7 \\ 2\end{array}\right)=21$ Paare von Analysengruppen auf Millimeterpapier gezeichnet. Werte, die aus den dabei entstehenden Punktwolken deutlich herausfielen - sog. Ausreißer -, wurden vor dem letzten Schritt der Regressionsanalyse (s. u.) eliminiert. In den nachfolgenden Darstellungen der Wertepaare $\left(f_{i} / q_{i}\right)$ wurde $f$ jeweils der Ordinate und $q$ der Abszisse zugeordnet (vgl. Abb. 2).

Diese Zuordnung soll keine eindeutige Abhängigkeit beschreiben, da ja die Untersuchung erst prüfen soll, ob eine solche Abhängigkeit vorliegt. Unter der Annahme, daß eine lineare Korrelation zwischen den Wertepaaren $\mathrm{f}$ und $\mathrm{q}$ besteht, wurden folgende Größen berechnet:

Der Korrelationskoeffizient r (Gleichung (4)), die Parameter a und $\mathrm{b}$ der beiden Regressionsgeraden (Gleichungen (5) und (6) bis (7)) $\quad$ il

$$
\begin{aligned}
r & =\frac{\left[f_{q}\right]-N^{-1}[f][q]}{\sqrt{\left(\left[q^{2}\right]-N^{-1}[q]^{2}\right)\left(\left[f^{2}\right]-N^{-1}[f]^{2}\right)}} \\
\bar{f} & =a_{f q}+b_{f q} q \text { und } \bar{q}=a_{q f}+b_{q q} q \\
a_{f q} & =\frac{[f]\left[q^{2}\right]-[q][f q]}{N\left[q^{2}\right]-[q]^{2}} \\
b_{f q} & =\frac{N[f q]-[q][f]}{N\left[q^{2}\right]-[q]^{2}}
\end{aligned}
$$

(Zur Berechnung von $a_{q}$ und $b_{q f}$ werden $q$ und $f$ in den Gleichungen (6) und (7) vertauscht.)

Zusätzlich zu diesen beiden Regressionsgeraden wurde noch eine mittlere Regressionsgerade $\tilde{f}=a_{a}^{p}+b_{q}^{p} \cdot \tilde{q}$ berechnet, deren Steigung $b_{a}^{p}$ das geometrische Mittel der beiden Regressionskoeffizientenb $_{\mathrm{fq}}$ und $1 / \mathrm{b}_{\mathrm{q} t}$ ist. Die riachfolgenden Formeln (8), (9) sind im Mathematischen Teil abgeleitet und begründet.

$$
\begin{aligned}
& b_{q}^{p}=\sqrt{\frac{N\left[f^{2}\right]-[f]^{2}}{N\left[q^{2}\right]-[q]^{2}}} \\
& a_{q}^{f}=\bar{f}-b_{q}^{p} \cdot \bar{q}
\end{aligned}
$$

Als Maß für den mittleren quadratischen Abstand der Punktwolke von der mittleren Regressionsgeraden dient die Funktion $s_{q}^{\mathfrak{f}}$ (Gleichung (10)). Die mittleren quadratischen Abstände der Punkte von der mittleren Regressionsgeraden in Richtung Ordinate und Abszisse beschreiben die Funktion $s_{\mathbb{P} / \mathbf{q}}$ und $s_{\mathbf{q} / \mathbf{q}}$ (Gleichungen (11) und (12)).

$$
\begin{aligned}
& s_{q}^{p}=\sqrt{\frac{\left[f_{i}-\tilde{f}_{i}\right]^{2}}{N-2}} \cdot \cos \alpha=s_{q / q}\left(\frac{1}{1+b_{q}^{f^{2}}}\right)^{2 / 2} \\
& \mathrm{~s}_{\mathrm{t} / \mathrm{q}}=\sqrt{\frac{\left[\mathrm{f}_{\mathrm{i}}-\tilde{\left.\tilde{\mathrm{f}}_{\mathrm{i}}\right]^{2}}\right.}{\mathrm{N}-2}} \\
& s_{q / s}=\sqrt{\frac{\left[q_{i}-\tilde{q}_{i j}\right]^{2}}{N-2}}
\end{aligned}
$$

\section{Ergebnisse}

Mit den Thrombokinasen der angegebenen Hersteller betrug die Wiederholbarkeit in Serie der Eichkurven 2-3 Relativ-Prozent. Die mit Plasmagemischen Gesunder an verschiedenen Tagen reproduzierten Eichkurven wichen maximal um 3-4 Norm-Prozent voneinander ab. Die Eichkurven der einzelnen Präparate weichen jedoch im Verlauf merklich voneinander $a b$ (Abb. 1).

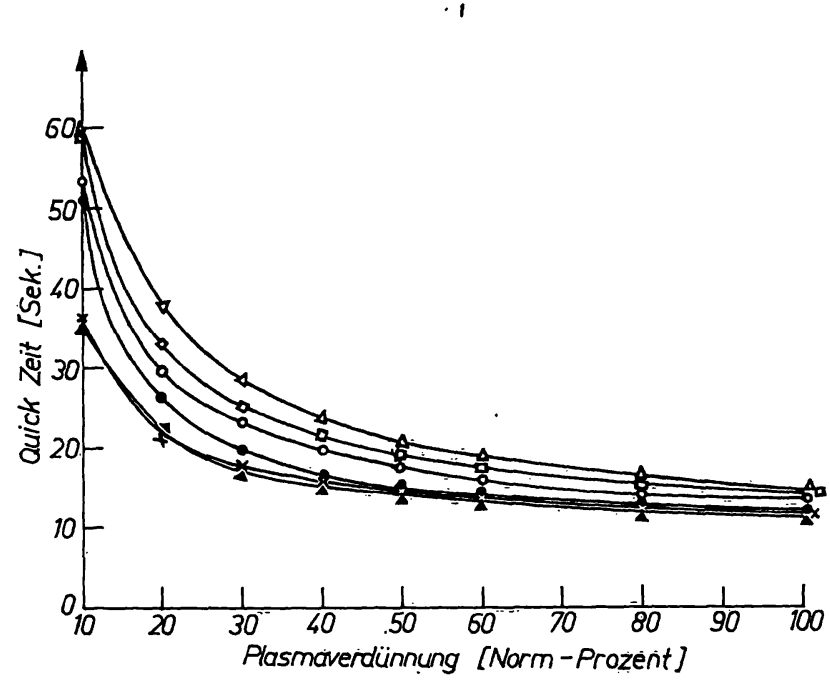

Abb. 1

Eichkurven für die Präparate der verschiedenen Hersteller. Diese wurden durch Verdünnung eines Plasma-Pools von 10 gesunden Proban-

$$
\begin{array}{ll}
\Delta-\Delta \text { Behring } & \bullet-\times \text { Dade } \\
\square-\square \text { Geigy } & \times-\times \text { Simplastin } \\
\circ \rightarrow 0 \text { La Roche } & \Delta-\Delta \text { Hyland] }
\end{array}
$$

Bei der gleichzeitigen Bestimmung des Quickwertes mit 7 verschiedenen Thrombokinase-Präparaten wurden im Einzelfall Abweichungen der Ergebnisse voneinander bis zu 25 Norm-Prozent beobachtet. Als Beispiel gibt die Abbildung 2 die Punktwolke des Vergleichs Simplastin/ Geigy wieder. Bildet man die Mittelwerte und Standardabweichungen einer jeden einzelnen Gruppe (Tab. 1),

\begin{tabular}{|c|c|c|c|c|}
\hline Thrombokinase & $\overline{\mathbf{x}}$ Norm \% & $\begin{array}{l}\text { Zahl der } \\
\text { Patienten }\end{array}$ & \pm s Norm \% & VK Relativ-\% \\
\hline Behring & 31,5 & 75 & 9.6 & 30,4 \\
\hline Geigy & 35,2 & 76 & 8,5 & 24,1 \\
\hline $\begin{array}{l}\text { Hoffmann- } \\
\text { La Roche }\end{array}$ & 34,0 & 76 & 11,1 & 32,7 \\
\hline $\begin{array}{l}\text { Simplastin= } \\
\text { Gödecke }\end{array}$ & 42,4 & 75 & 16,0 & 37,8 \\
\hline Dade & 44,8 & 76 & 18,0 & 40,1 \\
\hline Hyland & 40,2 & 75 & 13,9 & 34,5 \\
\hline $\begin{array}{l}\text { Thrombotest- } \\
\text { Nygård }\end{array}$ & 11,8 & 57 & 4,0 & 33,8 \\
\hline
\end{tabular}
so zeigt sich, daß hier eine über den individuellen Fall hinausgehende Regelmäßigkeit vorliegt: Die Mittelwerte desselben Patientenkollektivs variieren zwischen 31,5 und 44,8 Norm-Prozent. Die qualitativè Úberprüfung der - insgesamt 21 - Paarvergleiche, auf deren Abbildung verzichtet wurde, ergab besonders für das Präparat der Firma Dade eine auffallend schlechte Korrelation, ohne daß wir einen besonderen Anhaltspunkt

Tab. 1

Mittelwerte, Standardabweichungen und Variationskoeffizienten der beobachteten Quickwerte (Nicht bereinigte Rohdaten) 
Tab. 2

Korrelationskoeffizienten $\mathbf{r}$ der beobachteten Quickwerte (linke untere Hälfte) und Parameter und Streuungsmaße der optimalen Regressionsgeraden (rechte obere Hälfte der Tabelle)

\begin{tabular}{|c|c|c|c|c|c|c|c|c|c|c|c|c|c|}
\hline \multirow{2}{*}{$\frac{q \backslash f}{\text { Behring }}$} & \multirow{2}{*}{$\frac{\text { Behring }}{-}$} & \multicolumn{2}{|c|}{ Geigy } & \multicolumn{2}{|c|}{$\begin{array}{l}\text { Hoffmann- } \\
\text { La Roche }\end{array}$} & \multicolumn{2}{|c|}{ Simplastin } & \multicolumn{2}{|c|}{ Dade } & \multicolumn{2}{|c|}{ Hyland } & \multicolumn{2}{|c|}{ Thrombotest } \\
\hline & & $\begin{array}{r}+4,76 \\
69 \\
2,48\end{array}$ & $\begin{array}{l}1,013 \\
1,745 \\
2,45\end{array}$ & $\begin{array}{r}-3,07 \\
75 \\
6,53\end{array}$ & $\begin{array}{l}1,174 \\
4,23 \\
5,56\end{array}$ & $\begin{array}{r}-5,64 \\
67,74\end{array}$ & $\begin{array}{l}1,517 \\
3,71 \\
4,40\end{array}$ & $\approx \underset{76}{-8,7}$ & $\approx \underline{1,70}$ & $\begin{array}{c}-7,20 \\
7,31\end{array}$ & $\begin{array}{l}1,587 \\
3,90 \\
4,61\end{array}$ & $\begin{array}{c}-8,87 \\
3,06\end{array}$ & $\begin{array}{l}7,729 \\
2,475 \\
4,20\end{array}$ \\
\hline Geigy & 0,865 & & - & $\begin{array}{c}8,86 \\
60 \\
2,74\end{array}$ & $\begin{array}{l}0,817 \\
2,12 \\
3,36\end{array}$ & $\begin{array}{c}-19,8 \\
66 \\
11,5\end{array}$ & $\begin{array}{l}1,727 \\
5,77 \\
6,67\end{array}$ & $\approx-23,9$ & $\approx \underline{1,962}$ & $\begin{array}{c}-21,4 \\
74 \\
6,90\end{array}$ & $\begin{array}{l}1,743 \\
3,43 \\
3,96\end{array}$ & $\begin{array}{l}-9,5 \\
58 \\
2,85\end{array}$ & $\begin{array}{l}0,640 \\
2,40 \\
4,46\end{array}$ \\
\hline Hoffmann- & & \multirow{2}{*}{\multicolumn{2}{|c|}{0,910}} & & & 0,3 & 1,220 & \multirow{2}{*}{\multicolumn{2}{|c|}{$\begin{array}{c}\approx-5,1 \approx 1,485 \\
70 \\
(12,98)(7,25) \\
(8,13)\end{array}$}} & $-0,2$ & 1,236 & $-3,76$ & 0,506 \\
\hline La Roche & 0,866 & & & \multicolumn{2}{|c|}{-} & $\begin{array}{l}66 \\
5,34\end{array}$ & $\begin{array}{l}3,37 \\
4,35\end{array}$ & & & $\begin{array}{l}67 \\
4,62\end{array}$ & $\begin{array}{l}2,91 \\
3,74\end{array}$ & $\begin{array}{l}56 \\
2,94\end{array}$ & $\begin{array}{l}2,62 \\
5,81\end{array}$ \\
\hline Simplastin & 0,749 & \multicolumn{2}{|c|}{0,839} & \multicolumn{2}{|c|}{0,758} & \multicolumn{2}{|c|}{-} & $\approx-\frac{2,6}{-}$ & $\approx \underline{1,118}$ & $\begin{array}{c}-3,0 \\
69 \\
6,41\end{array}$ & $\begin{array}{l}1,056 \\
4,40 \\
6,07\end{array}$ & $\begin{array}{l}-3,0 \\
56 \\
2,73\end{array}$ & $\begin{array}{l}0,391 \\
2,54 \\
6,97\end{array}$ \\
\hline Dade & 0,651 & \multicolumn{2}{|c|}{0,765} & \multicolumn{2}{|c|}{0,698} & \multicolumn{2}{|c|}{0,639} & \multicolumn{2}{|c|}{ - } & $\begin{array}{l}+2,6 \approx \\
\underline{75}\end{array}$ & $\approx \underline{0,840}$ & $\approx-1,0$ & $\approx \underline{0,283}$ \\
\hline Hyland & 0,798 & \multicolumn{2}{|c|}{0,894} & \multicolumn{2}{|c|}{0,910} & \multicolumn{2}{|c|}{0,819} & \multicolumn{2}{|c|}{0,795} & & - & $\begin{array}{r}-1,63 \\
38,03\end{array}$ & $\begin{array}{l}0,369 \\
1,90 \\
5,50\end{array}$ \\
\hline Thrombotest & 0,628 & \multicolumn{2}{|c|}{0,797} & \multicolumn{2}{|c|}{0,742} & \multicolumn{2}{|c|}{0,700} & \multicolumn{2}{|c|}{0,743} & \multicolumn{2}{|c|}{0,865} & \multicolumn{2}{|r|}{ 一 } \\
\hline
\end{tabular}

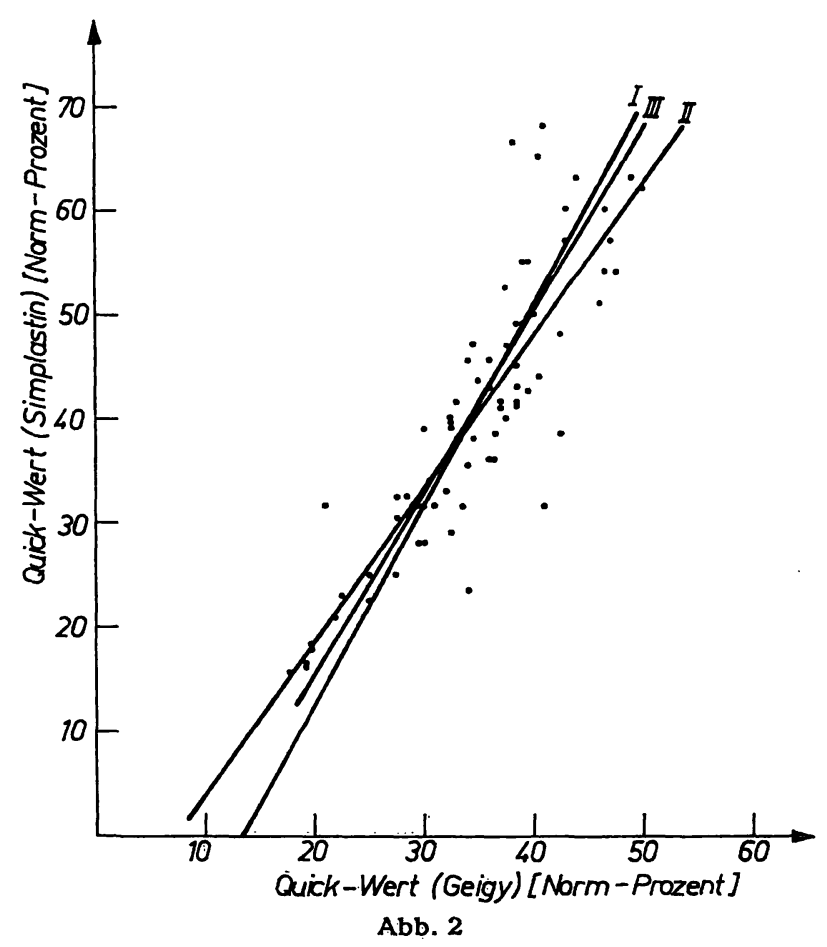

Vergleich von Quickwerten: Bestimmung mit Thrombokinase Simplastin (Gödecke) und Thrombokinase (Geigy).

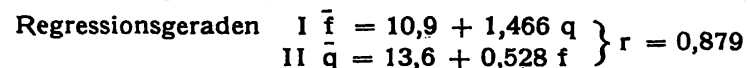

$$
\text { III } \begin{aligned}
\tilde{\mathbf{f}} & =a+b_{b}^{x} \tilde{\mathbf{q}} \\
\tilde{\mathbf{f}} & =-19,8+1,727 \tilde{\mathbf{q}}
\end{aligned}
$$$$
\text { (mittlere Regressionsgerade) }
$$

dafür finden konnten. Aus diesem Grunde haben wir auf die Wiedergabe einiger Ergebnisse, die an diesem Präparat gewonnen wurden, verzichtet.

\section{Regressionsanalyse}

Die Korrelationșkoeffizienten der 21 Kombinationen von Thrombokinase-Präparaten (T'ab. 2, linke untere Hälfte) liegen zwischen 0,628 and 0,910. Dieses Ergebnis ist auch im schlechtesten Fall hochsignifikant $(p<0,01)$. Die klassische Regressionsanalyse ergab jedoch in den meisten Fällen zwei merklich voneinander abweichende Geraden. Für klinische Zwecke wird jedoch eine einzige Regressionsgerade zum Umrechnen von Wertepaaren in beiden Richtungen benötigt. (Auf die Besonderheiten des statistischen Modells der bivariaten Regressionsanalyse wird in der Diskussion eingegangen.)

Die Parameter dieser "mittleren Regressionsgeraden“" sind in der rechten oberen Hälfte von Tabelle 2 angegeben. Die Abweichungen vom "Idealfall" $(a=0$, $\mathrm{b}=1,0$ ) sind $z$. $T$. ganz erheblich.

Die Werte der mittleren Regressionsgeraden erlauben das Aufstellen einer Vergleichstabelle (Tab. 3), mit deren Hilfe man die mit verschiedenen Thrombokinasen gewonnenen Quickwerte ineinander umrechnen kann. Die Tabelle 3 bezieht sich auf eins der angegebenen Präparate, ist aber beliebig umstellbar. Die Umrechnung ist nur innerhalb der angegebenen Grenzen vertretbar, weil nur für diesen Bereich genügend Meßpunkte vorliegen.

\section{Diskussion}

Die Thromboplastinzeiten, die mit verschiedenen Präparaten ermittelt wurden, variieren auch in unseren Untersuchungsteihen erheblich (1-4). Die im Handel angebotenen Präparate unterscheiden sich nach Herkunft (Species und Organ), Präparation, Eichung und ihrer Empfindlichkeit gegen Faktor VII, X und IX $(5,6)$. Es wäre erstaunlich, wenn sich alle diese genannten Effekte gegenseitig so aufheben würden, $\mathrm{da} ß$ übereinstimmende Resultate, selbst bei einheitlicher Technik, erzielt werden

Tab. 3

\begin{tabular}{|c|c|c|c|c|c|c|}
\hline Behring & Geigy & $\begin{array}{l}\text { H.- La } \\
\text { Roche }\end{array}$ & $\underset{\text { plastin }}{\text { Sim- }}$ & Dade & Hyland & $\begin{array}{c}\text { Thrombo- } \\
\text { test }\end{array}$ \\
\hline $\begin{array}{l}10,0 \\
12,5 \\
15,0 \\
17,5 \\
20,0 \\
22,5 \\
25,0 \\
27,5 \\
30,0 \\
32,5 \\
35,0 \\
37,5 \\
40,0 \\
42,5 \\
45,0 \\
47,5 \\
50,0\end{array}$ & $\begin{array}{l}20,0 \\
22,5 \\
25,0 \\
27,6 \\
30,1 \\
32,6 \\
35,2 \\
37,7 \\
40,2 \\
42,7 \\
45,3 \\
47,8 \\
50,3 \\
52,9 \\
55,4\end{array}$ & $\begin{array}{l}20,4 \\
23,3 \\
26,3 \\
29,2 \\
32,2 \\
35,1 \\
38,0 \\
41,0 \\
43,9 \\
46,8 \\
49,8\end{array}$ & $\begin{array}{r}9,5 \\
13,3 \\
17,1 \\
20,9 \\
24,7 \\
28,5 \\
32,3 \\
36,1 \\
39,9 \\
43,7 \\
47,5 \\
51,2 \\
55,0 \\
58,8 \\
62,6 \\
66,4 \\
70,2\end{array}$ & $\begin{array}{r}8,3 \\
12,6 \\
16,8 \\
21,0 \\
25,3 \\
29,6 \\
33,8 \\
38,0 \\
42,3 \\
46,6 \\
50,8 \\
55,0 \\
59,3 \\
63,6 \\
67,8\end{array}$ & $\begin{array}{l}16,6 \\
20,6 \\
24,5 \\
28,5 \\
32,5 \\
36,4 \\
40,4 \\
44,4 \\
48,3 \\
52,3 \\
56,3 \\
60,2 \\
64,2\end{array}$ & $\begin{array}{r}(2,1) \\
(3,9) \\
5,7 \\
7,5 \\
9,4 \\
11,2 \\
13,0 \\
14,8 \\
16,6 \\
18,5 \\
20,3\end{array}$ \\
\hline
\end{tabular}

Umrechnen von Quickwerten (Norm-Prozent) ineinander 
könnten. Systematische Fehler ergeben sich auch bei der Umrechnung von Zeit auf Norm-Prozent mit einer Verdünnungseichkurve (3). Während durch die CumarinBehandlung die Faktoren II, VII, IX und X (7) betroffen sind, werden ja zur Herstellung der Eichkurven alle Faktoren verdünnt. Aus diesem Grund sollte auch dazu übergegangen werden, den Thromboplastinzeitquotienten

$$
\text { (Thromboplastinzeit des Normalplasmas })
$$

mit anzugeben (8).

$\mathrm{Da}$ mit einer Standardisierung der Präparate (9) in absehbarer Zeit nicht gerechnet werden kann, müssen - wenn auch unter Vorbehalten - Umrechnungsfaktoren für die verschiedenen Präparate ermittelt werden. Die vorliegende Berechnung kann sich nur auf den therapeutischen Bereich, nicht aber auf den Normalbereich beziehen, weil nur Untersuchungsmaterial von Patienten vorliegt, die mit Cumarin eingestellt wurden. Wir möchten zu Vergleichsuntersuchungen anregen und schlagen eine einfache Methode zur Umrechnung der Gerinnungsanalysen vor.

Bei den nachfolgenden Überlegungen wurde unterstellt, daß zwischen den zu vergleichenden Variablen $f$ und $\mathrm{q}$ ein lineares Verhältnis vorliegt. Die klassische Regressionṡanalyse macht beim Vergleich zweier Meßreihen die Annahme, daß der zufallsbedingte Fehler der einen ( $y$ ) wesentlich größer als der der anderen $(x)$ ist und kommt damit $z u$ relativ einfachen Formeln. Im vorliegenden Problem muß jedoch angenommen werden, $\mathrm{da} B$ beide der $z u$ vergleichenden Variablen mit einem annähernd gleich großen und deutlich in Erscheinung tretenden zufallsbedingten Fehler behaftet sind. Wendet man die klassische Regressionsanalyse an, so erhält man zwei voneinander abweichende Geraden, je nachdem, welcher Variablen $f$ oder $q$ man die größere statistische Variabilität zuordnet (Gerade I und II in Abb. 2). Beide Geraden schneiden sich im Punkt $(\bar{f} / \bar{q})$, dessen Koordinaten den arithmetischen Mitteln der Variablen $f_{i}$ und $q_{i}$ entsprechen. Aus praktischen Gründen sollten diese beiden Geraden jedoch durch eine einzige Gerade ersetzt werden, die nachfolgend die „mittlere Regressionsgerade" genannt wird. Die mathematischen Details bitten wir den Ableitungen im mathematischen Teil zu entnehmen. Wie sich zeigen läßt, legt man eine solche mittlere Regressionsgerade, die man unterschiedlich definieren kann, zweckmäßigerweise durch den Punkt $\overline{(f / q}$, d. h. den Schnittpunkt der Regressionsgeraden. Als Steigung der mittleren Regressionsgeraden $b_{q}^{p}$ wählen wir das geometrische Mittel der Steigungen der Regressionsgeraden. Diese Definition zeigt eine gewisse Ähnlichkeit zu dem vielbenutzten Produkt-Moment-Korrelationskoeffizienten $r$, der ebenfalls ein geometrisches Mittel dieser Steigungen ist.

Diese Definition führt überdies zu recht einfachen Formeln zum Berechnen von $b_{\mathrm{q}}^{\mathfrak{q}}$.

Um die Abweichung der Punktwolke von der mittleren Regressionsgeraden quantitativ zu beschreiben, werden die Streuungsmaße $s_{q}^{q}, s_{\ell / q}$ und $s_{q / 2}$ definiert. Diese geben einen Anhalt für den mittleren quadratischen Abstand der Punkte zur mittleren Regressionsgeraden senkrecht auf diese und in Richtung der beiden Koordinaten. Je kleiner diese Streuungsmaße sind, desto besser ist die Korrelation zwischen $f$ und $q$.

In der Tabelle 1 zeichnen sich 3 Gruppen von Präparaten ab: 1. Behring, Geigy, La Roche; 2. Simplastin, Dade, Hyland; und 3. Thrombotest. Es ergaben sich in der zweiten Gruppe höhere Mittel lwerte als in der ersten. Die Sonderstellung des Thrombotests ist bekannt und wird bei der Einstellung der Patienten berücksichtigt (10). Es wurden nun die mit verschiedenen Thrombokinasen ermittelten Werte ineinander umgerechnet. Legt man die Streuung der Meßpunkte um die mittlere Regression und die Korrelationskoeffizienten zugrunde, so passen die Paare Behring/Geigy, Geigy/La Roche und Thrombotest/Hyland am günstigsten zueinander. Das Umrechnen von Thrombokinase-Dade-Ergebnissen empfehlen wir wegen der sehr schlechten Korrelation (vgl. das Beispiel La Roche/Dade, Tab. 2) nicht.

\section{Schlußfolgerungen}

1. Gerinnungsanalysen, z. B. Quickwerte, sollten mit exakten Angaben über das verwendete Reagenz dokumentiert werden.

2. Das Ergebnis sollte sowohl in Norm-Prozent wie auch in einer zweiten Maßeinheit, z. B. als Quickzeitquotient, ausgedrückt werden.

3. Mit verschiedenen Reagenzien gewonnene Werte lassen sich näherungsweise ineinander umrechnen. Die einzelnen Kombinationen von Präparaten unterscheiden sich merklich in der Sicherheit der Umrechnung zueinander.

\section{Mathematischer Teil}

Ableitung der verwendeten Formeln zur bivariaten RegresGleicbungen der klassischen Regressionsgeraden

Zwei Populationen von Variablen $f_{i}$ und $q_{i}$ stehen in einem linearen Zusammenhang miteinander. Beide sind jedoch mit einem merklichen zufallsbedingten Fehler behaftet. Trägt man alle $f_{1}$ und $q_{i}$ in ein Koordinatenschema ein, so ergibt sich eine Punktwolke wie in $A b$ bildung 2 , die sich mehr oder weniger gut einer noch $z u$ berechnenden Geraden $\tilde{f}=a_{q}^{p}+b_{q}^{p} \tilde{q}$ anschmiegt. Die klassische Regressionsanalyse nimmt vereinfachend an, $\mathrm{daß}$ der Meßfehler der einen Variablen beträchtlich größer ist als der Meßfehler der zweiten Variablen (vgl. l. c. (11)). Nimmt man dies jeweils für $f_{1}$ und $q_{1}$ an, und fordert, $\mathrm{da} \beta$ die Summe der Abstandsquadrate aller Punkte in Richtung $f$ oder $q$ auf die Ausgleichsgerade ein Minimum wird, so erhält man folgende Ausgangsbedingungen:

$$
\begin{aligned}
& \mathrm{Q}_{1}=\left[\mathrm{df}_{1}^{2}\right]=\left[\left(\overline{\mathrm{f}}_{1}-\mathrm{f}_{1}\right)^{2}\right]=\mathrm{MIN} \\
& \mathrm{Q}_{2}=\left[\mathrm{dq}_{1}{ }^{2}\right]=\left[\left(\overline{\mathrm{q}}_{1}-\mathrm{q}_{1}\right)^{2}\right]=M I N
\end{aligned}
$$


Die Parameter a und $b$ der Regressionsgeraden

$$
\bar{f}_{1}=a_{q}+b_{1} q \text { und } \bar{q}_{1}=a_{q}+b_{q} f
$$

können durch Bilden der partiellen Ableitungen $\frac{\partial Q}{\partial a}$ und $\frac{\partial Q}{\partial b}$, die gleich Null sein müssen, abgeleitet werden, was hier nur angedeutet werden soll. Die ausführliche $\mathrm{Ab}$ leitung ist bei ZuRMüHL (11) angegeben. Man erhält in beiden Fällen ein System von sog. Normalgleichungen:

$$
\begin{array}{ll}
N a_{f}+\left[q_{1}\right] b_{t}=\left[f_{1}\right] & N a_{q}+\left[f_{1}\right] b_{q}=\left[q_{1}\right] \\
{\left[q_{1}\right] a_{t}+\left[q_{1}^{2}\right] b_{t}=\left[f_{1} q_{1}\right]} & {\left[f_{1}\right] a_{q}+\left[f_{1}^{2}\right] b_{q}=\left[q_{1} f_{1}\right]}
\end{array}
$$

$\mathrm{b}$ errechnet sich in beiden Fällen als Quotient zweier Determinanten:

$$
\begin{aligned}
& b_{\mathrm{f}}=\mathrm{D}_{\mathrm{bf}} / \mathrm{D}_{\mathrm{f}} \\
& \text { mit } D_{\mathrm{vr}}=\left|\begin{array}{ll}
\mathrm{N} & {\left[\mathrm{f}_{1}\right]} \\
{\left[q_{1}\right]} & {\left[f_{1} q_{1}\right]}
\end{array}\right| \\
& \mathrm{D}_{\mathrm{z}}=\left|\begin{array}{ll}
\mathrm{N} & {\left[\mathrm{q}_{\mathbf{1}}\right]} \\
{\left[\mathbf{q}_{\mathbf{1}}\right]} & {\left[\mathbf{q}_{1}{ }^{2}\right]}
\end{array}\right| \\
& \begin{aligned}
b_{q} & =D_{b q} / D_{q} \\
D_{b q} & =\left|\begin{array}{ll}
N & {\left[q_{1}\right]} \\
{\left[f_{1}\right]} & {\left[q_{1} f_{1}\right]}
\end{array}\right| \\
D_{q} & =\left|\begin{array}{ll}
N & {\left[f_{1}\right]} \\
{\left[f_{1}\right]} & {\left[f_{1}^{2}\right]}
\end{array}\right|
\end{aligned}
\end{aligned}
$$

Analog lassen sich $a_{\&}$ und $a_{q}$ aus den Normalgleichungen ableiten, worauf hier verzichtet werden soll.

Wie Abbildung 2 zeigt (Gerade I und II), weichen die beiden Regressionsgeraden $\bar{f}_{i}$ und $\bar{q}_{1}$ merklich voneinander ab. Als $\mathrm{MaB}$ für diese Abweichung gibt man den Korrelationskoeffizienten $\mathrm{r}$ an, der - unbeschadet anderer Definitionen - als das geometrische Mittel der beiden Regressionskoeffizienten $b_{\ell}$ und $b_{q}$ aufgefaßt werden kann (12):

$$
r=\sqrt{b_{f} b_{q}} \quad|r| \leqq 1
$$

\section{Steigung $b_{q}^{q}$ der mittleren Regressionsgeraden}

Zwei Regressionsgeraden für eine Fragestellung sind eine unbefriedigende Lösung. Es wird nachfolgend versucht, diese beiden Geraden $\overline{\mathrm{f}}_{1}$ und $\overline{\mathrm{q}}_{1}$ durch eine Gerade zu ersetzen:

$$
\tilde{f}_{1}=a_{q}^{q}+b_{q}^{q} \tilde{q}
$$

Als Kriterien für diese "mittlere Regressionsgerade“ werden für die Steigung $b_{q}^{p}$ das geometrische Mittel der beiden Regressionskoeffizienten und für den Koordinatenabschnitt $a_{\mathbb{q}}^{q}$ eine Minimumbedingung vorgeschlagen.

Die beiden Regressionsgeraden lauten:

$$
\begin{aligned}
\bar{f}_{1_{1}}=a_{q}+b_{1} q_{1} \text { und } \bar{q}_{1}=a_{q}+b_{q} f_{1_{2}} \\
\text { oder } f_{i_{2}}=-\frac{1}{b_{q}} a_{q}+\frac{1}{b_{q}} \bar{q}_{1}
\end{aligned}
$$

Das geometrische Mittel der beiden Steigungen ist:

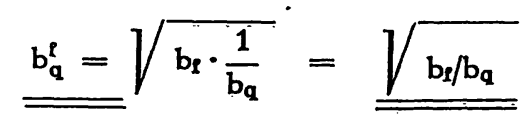

Setzt man nun die im vorangehenden Abschnitt angegebenen Gleichungen für $b_{\mathbf{q}}$ und $b_{q}$ ein, so erhält man eine sehr einfache Rechenvorschrift zur Ermittlung von $b_{q}^{p}$ :

$$
\left(b_{q}^{f}\right)^{2}=\frac{b_{q}}{b_{q}}=\frac{D_{b q} / D_{q}}{D_{b q} / D_{q}}=\frac{\left|\begin{array}{ll}
N & {\left[f_{1}\right]} \\
{\left[q_{1}\right]} & {\left[f_{1} q_{1}\right]}
\end{array}\right|}{\left|\begin{array}{ll}
N & {\left[q_{1}\right]} \\
{\left[q_{1}\right]} & {\left[q_{1}^{2}\right]}
\end{array}\right|^{-1}}
$$

$$
\begin{aligned}
&\left(b_{q}^{p}\right)^{2}=\frac{\left|\begin{array}{ll}
N & \left|f_{1}\right| \\
\left|f_{1}\right| & \left|f_{1}^{2}\right|
\end{array}\right|}{\left|\begin{array}{ll}
N & {\left[q_{1}\right]} \\
{\left[q_{1}\right]} & {\left[q_{1}^{2}\right]}
\end{array}\right|}=\frac{N \cdot\left[f_{1}^{2}\right]-\left[f_{1}\right]^{2}}{N \cdot\left[q_{1}^{2}\right]-\left[q_{1}\right]^{2}} \\
& b_{q}^{p}=\sqrt{\frac{N \cdot\left[f_{1}^{2}\right]-\left[f_{1}\right]^{2}}{N \cdot\left[q_{1}^{2}\right]-\left[q_{1}\right]^{2}}}
\end{aligned}
$$

\section{Ordinatenabschnitt $\mathrm{a}_{a}^{\mathrm{q}}$ der mittleren Regressionsgeraden}

Wie nachstehend gezeigt werden soll, geht eine Gerade $f=a+b q$, die die Minimumbedingung $Q=\left[\mathrm{d}_{1}{ }^{2}\right]$ $=$ MIN erfüllt, durch den Punkt (f/q). Als $d_{1}$ werden die Abstände der Meßpunkte senkrecht zur Geraden definiert.

$$
\begin{aligned}
& d_{1}=d f_{1} \cos \alpha=\left(a+b q_{1}-f_{1}\right) \cos \alpha \\
& Q=\left[a+b q_{1}-f_{1}\right]^{2} \cdot \cos ^{2} \alpha=\left[a+b q_{1}-f_{1}\right]^{2}\left(\frac{1}{1+b^{2}}\right)
\end{aligned}
$$

Die partielle Ableitung von $Q$ nach a liefert die Gleichung

$$
\begin{aligned}
& \frac{\partial Q}{\partial a}=2\left[a+b q_{1}-f_{1}\right]^{2} \cdot\left(\frac{1}{1+b^{2}}\right)=0 \\
& N a+b\left[q_{1}\right]-\left[f_{1}\right]=0 \\
& a=-b N^{-1}\left[q_{1}\right]+N^{-1}\left[f_{1}\right] \\
& a_{q}^{r}=\bar{f}-b_{q}^{r} \bar{q}
\end{aligned}
$$

Damit sind die Parameter $a_{q}^{q}$ und $b_{q}^{\mathfrak{q}}$ der mittleren Regressionsgeraden festgelegt. Für die Steigung $b$ ließe sich natürlich auch eine andere als die angegebene Definition finden, z. B. die soeben benutzte Minimumbedingung. Jedoch sind die dann resultierenden Formeln komplizierter und erfordern größeren Rechenaufwand, der sich im vorliegenden Fall unseres Erachtens nicht lohnt.

\section{Streuungsmaße der mittleren Regressionsgeraden}

Die mittlere Abweichung der Punktwolke $\left(f_{i} / q_{1}\right)$ von der mittleren Regressionsgeraden wird aus den Fehlerquadratsummen $\mathrm{Q}$ berechnet. Die mittleren Abweichungen senkrecht zur Regressionsgeraden werden mit ${ }_{q}^{?}$, die in Richtung der Ordinate mit $\mathrm{f} / \mathrm{q}$ und die in Richtung Abszisse mit $q / f$ indiziert. Die Fehlerquadratsummen lauten also:

$$
\begin{aligned}
& \mathrm{Q}_{\mathrm{q}}^{\mathrm{q}}=\left[\mathrm{d}_{1}{ }^{2}\right]=\left[\mathrm{df}_{\mathrm{1}}{ }^{2}\right]\left(\frac{1}{1+\mathrm{b}^{2}}\right) \\
& \mathrm{Q}_{\ell / \mathrm{q}}=\left[\mathrm{df}_{\mathrm{1}}{ }^{2}\right] \\
& \mathrm{Q}_{\mathrm{q} / \mathrm{q}}=\left[\mathrm{dq}_{\mathrm{i}}{ }^{2}\right]
\end{aligned}
$$

Die mittleren quadratischen Abweichungen werden hieraus definiert:

$$
\begin{aligned}
& s_{\mathbf{q}}^{p}=\sqrt{\frac{Q_{q}^{p}}{N-2}}=s_{t / q} \cdot \cos \alpha \\
& s_{\ell / q}=\sqrt{\frac{Q_{\ell / Q}}{N-2}} \\
& s_{\mathbf{q} / \mathbf{s}}=\sqrt{\frac{\mathrm{Q}_{\mathbf{q} / \mathbf{s}}}{\mathrm{N}-2}}
\end{aligned}
$$

Die so definierten Streuungsparameter $\mathbf{s}$ sind formal den Streuungsmaßen $\mathbf{s}_{\mathbf{y} . \mathbf{x}}$ der klassischen Regressionsgeraden ähnlich, aber nicht mit diesen identisch (vgl. l.c. (12)). 
Ein Rechenprogramm zum Berecbnen der mittleren Regressionsgeraden fir den Tiscbrecbner Olivetti Programm 101

\section{Bescljreibung des Programms}

Das Programm steht auf 2 Magnetkartenseiten. Im ersten Teil werden alle Meßpunkte $\left(\bar{f}_{1} / \bar{q}_{1}\right)$ eingegeben. Eine Korrektur inkorrekt eingegebener Werte ist mög- lich. Nach Ausdruck von Zwischensummen werden die Parameter $a_{q}^{f}$ und $b_{q}^{q}$ der mittleren Regressionsgeraden sowie die Koordinaten des arithmetischen Mittelpunktes $(\overline{\mathrm{f}} / \overline{\mathrm{q}})$ ausgedruckt.

Im zweiten Teil werden die Meßpunkte erneut eingegeben und hieraus die Streuungsparameter $s_{q}^{\ell}, s_{\ell / q}$ und $s_{\mathbf{q} / \text { }}$ berechnet.
Ablaufbeschreibung

Seite 1 der Magnetkarte

1. Stellung des Dezimalstellenrades: 4

2. Netzschalter ein. Löschtaste bedienen. Programmkarte Seite 1 durchlaufen lassen.

\section{Dateneingabe}

4. Eingabe ' $f_{i}$ ', 'S'

Schritte 4 und 5 mit allen Wertepaaren $f_{1} / q_{1}$ ausführen. Bei Eingabefehlern: Schritt 6 . Nach Eingabe aller Daten: Schritt 11 ausführen.

Fehlerkorrektur

6. ' $\mathrm{CZ}$ '

7. 2 Zeilenwechsel

8. Eingabe ' $f_{i}$, falsch'

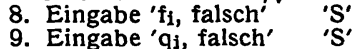

10. Eingabe ' $q_{i j}$, falsch'

2 Zeilenwechsel Rücksprung nach 4. Berechnen von $a_{q}^{p}$ und $b_{q}^{p}$

11. ' $Z$ '

12. Ausdruck von $Z$ wischensummen und Ergebnissen

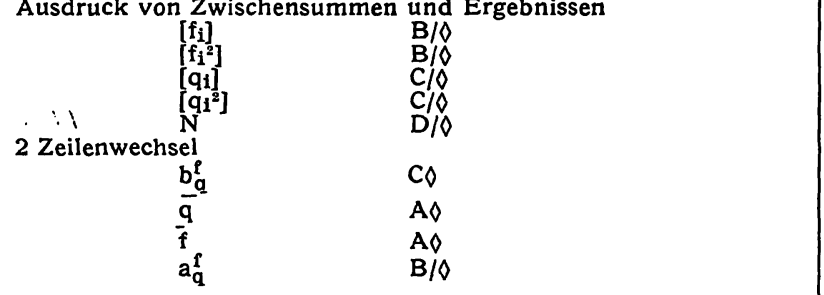

Ablaufbeschreibung

Seite 2 der Magnetkarte

1. Stellung des Dezimalstellenrades: 4

2. Nicht die Löschtaste drücken. Programmkarte Seite 2 durchlaufen lassen.

4. Ausdruck

$$
\begin{array}{ll}
a_{q}^{p} & B / \delta \\
b_{q}^{p} & c \diamond
\end{array}
$$

2 Zeilenwechsel

5. Eingabe ' $\mathrm{f}_{\mathbf{i}}{ }^{\prime}$ ' ' $\mathrm{S}$ '

Entscheidung

Eingabe richtig? Schritt 6 ausführen

Eingabe falsch? Schritt 5 wiederholen

6. 'Y'

2 Zeilenwechse

Dann das nächste Wertepaar eingeben: Schritt 5

Berechnung der Streuungsparameter

7. ' $\mathrm{Z}$ '

8. Nach 1 Zeilenwechsel Ausdruck der Zwischensummen:

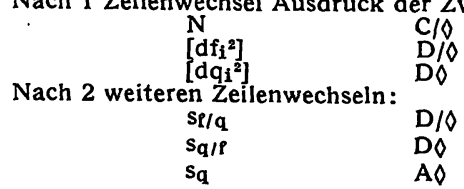

\section{Befehisliste}

Die Programmbefehle sind spaltenweise/von oben nach unten zu lesen

Programmkärte, S. 1

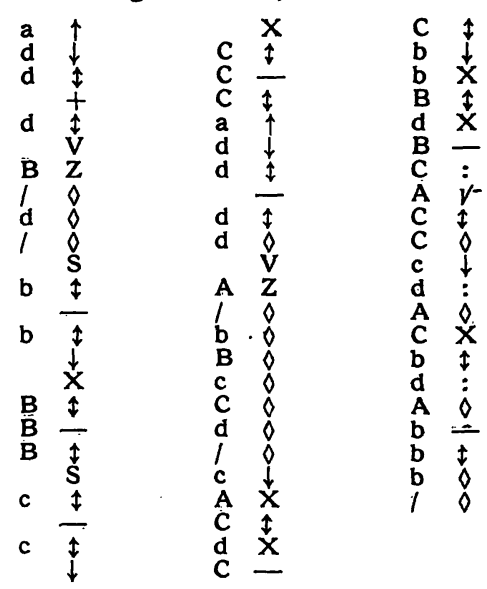

Programmkarte, S. 2

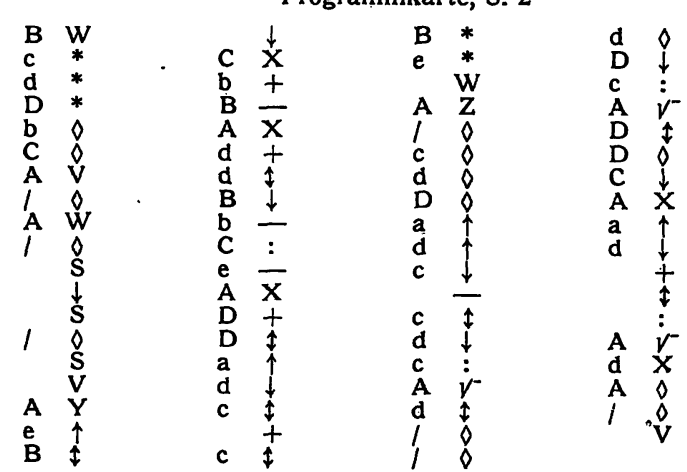

\section{Literatur}

1. Biggs, R., Thrombos. Diathes. haemorr., Suppl. 17, 303 (1965). 2. Brggs, R. und Denson, K. W. E., Thrombos. Diathes. haemorr., Suppl. 20, 345 (1966). - 3. Holzknecht, F., Wien. med. Wschr. 78, 73 (1966). - 4. Stormorken, H., Symposion über Indikationen und Uberwachung der peroralen Antikoagulantien-Therapie. 30. September 1966 in Heidelberg. - 5. Loelinger, A., Schweiz. med. Wschr. 639 (1958). - 6. Verstruire, M., C. VerMYLEN und J. VANDENSRouTh, Acta med. Scand. 163, 105 (1959).-
7. Popoccenic, S., Med. Klin. 61, 1960 (1966). - 8. Biggs, R., Thrombos. Diathes. haemorr., Suppl. 21, 433 (1967). - 9. DENsoN, K. W. E., Thrombos. Diathes. haemorr., Suppl. 21, 437 (1967). - 10. KaYser, J. W., Brit. Med. J. 1514 (1963). - 11. ZuRмüнL, R., Praktische Mathematik. Springer Verlag Berlin, 5. Aufl. (1965). - 12. Fuchs, G., Mathematik für Mediziner und Biologen. Springer Verlag Berlin (1969).

Dr. R. Averdunk 1000 Berlin 45 Hindenburgdamm 30 


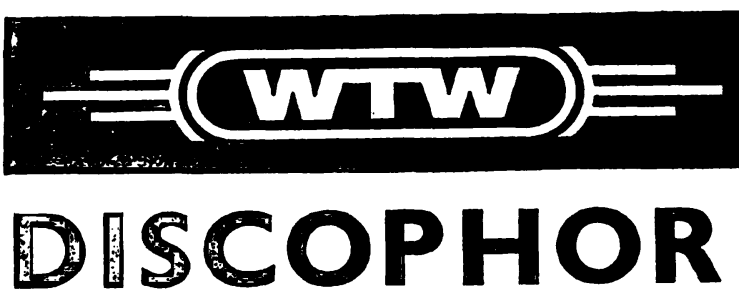

der programmierbare Disk-ElektrophoreseAutomat EA 100 fährt wahlweise

- präparativ

- oder

- analytisch

und liefert bei präparativer Verwendung automatisch
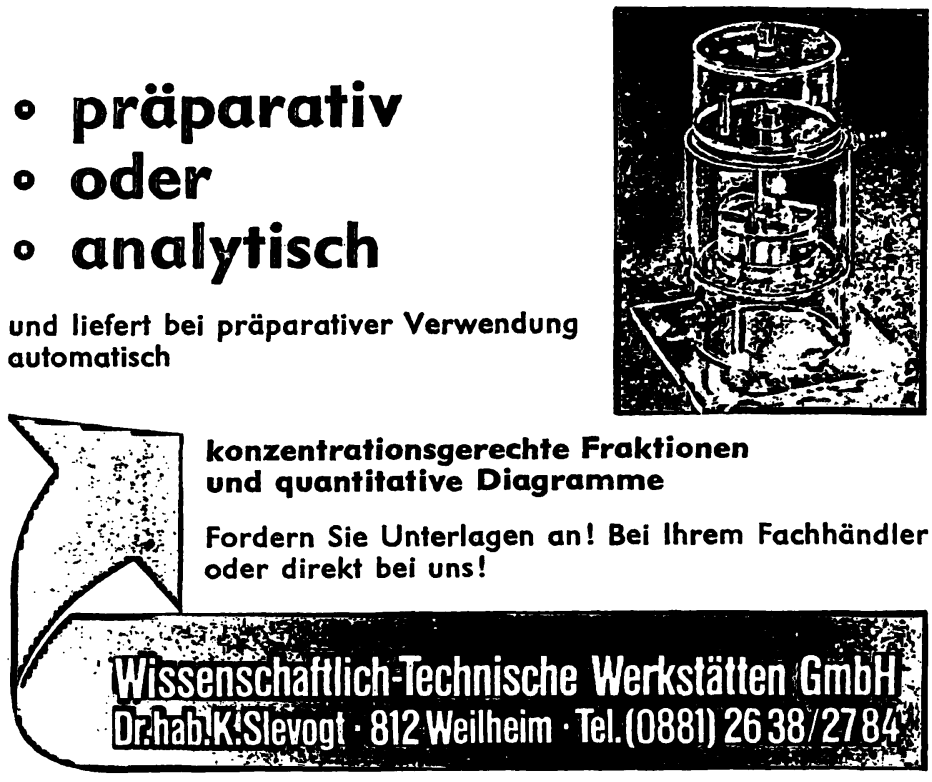

Verkaufbüros: Essen, Lönsberg 22, Tel.:510019

Dusslingen, W. Bohn, Farrenbergweg_5, Tel. 071 28/7809

Hagen, Hestertstraße 64, Tel. 45857

Bad Nauheim, Frankfurter Str. 39, Tel. 4860

Auf der Analytica sind wir in Halle 2 Stand 2204 vertreten.

\section{Asmus}

\section{Einführung \\ in die Höhere Mathematik und ihre Anwendungen}

Ein Hilfsbuch für Chemiker, Physiker und andere Naturwissenschaftler

Von Prof. Dr. Erik Asmus

5. Auflage. Oktav. Mit 184 Abbildungen. XII, 410 Seiten. 1969. Plastik flexibel DM 24,-

Wir wollen mit ganz besonderem Nachdruck darauf hinweisen, daß hier ein Buch vorliegt, das so sehr in der Sprache des Naturwissenschaftlers geschrieben wird, $\mathrm{da} ß$ es für den Biologen, Chemiker oder Physiker in ganz besonderem Maße lesbar wird. Vom Stoff her vermittelt das Buch ausführlich und an Hand sehr vieler Beispiele aus der Praxis des Naturforschers die Differentialrechnung, und die Integralrechnung.

Die Natur, Stuttgart

Walter de Gruyter \& Co $\cdot$ Berlin

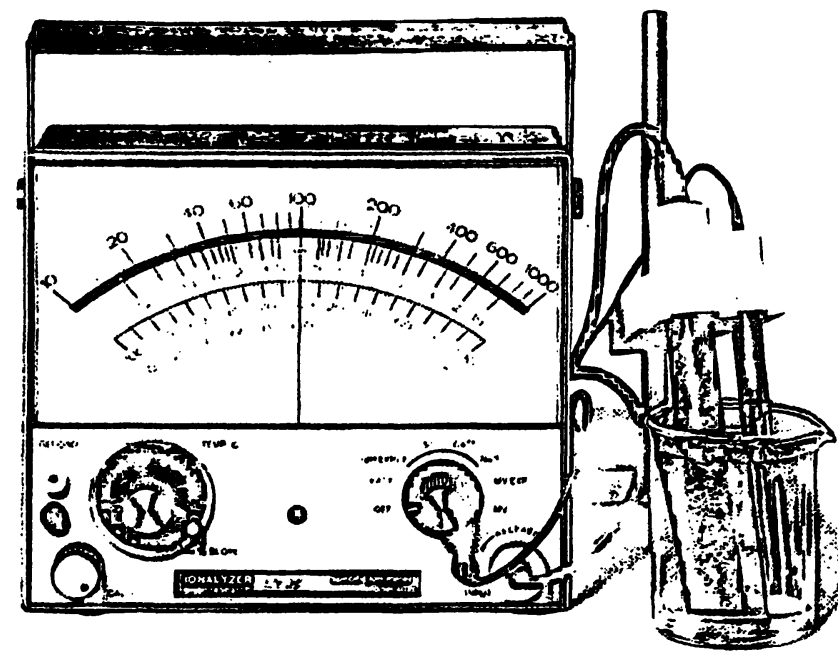

\section{Orion}

\section{Ionenselektive Elektroden}

Eine neue Technologie zur ionenselektiven Analyse flüssiger Medien. Die Analysensysteme bestehen aus speziellen Elektroden und elektronischen Digital- bzw. Analoggeräten. Es sind damit Einzel- und Simultanbestimmungen von z.B. $\mathrm{F}^{-}, \mathrm{Cl}^{-}, \mathrm{CN}^{-}$, $\mathrm{Na}^{+}, \mathrm{Ca}^{++}, \mathrm{Cu}^{++},\left(\mathrm{Ca}^{++}+\mathrm{Mg}^{++}\right)$ möglich. Das Verfahren ist leicht zu handhaben und methodisch erprobt.

\section{Colora Messtechnik GmbH} 7073 Lorch/Württ., Postfach 5 T (07172) 6041, FS 07-248 886

Technische Büros (Verkauf und Kundendienst): 1000 Berlin 30, Kurfürstenstraße 84, T 135200 2000 Hamburg 19, Osterstraße 63, T 4006 06, FS 02-12947 3000 Hannover, An der Tiefenriede 45, T 884500 4000 Düsseldorf, Kronprinzenstr. 62, T 17860 , FS 08-587 253 6000 Frankfurta.M.,Röderbergweg 4-6,T 4460 31, FS 04-11216 8000 München 2, Dachauer Straße 175, T 5169858

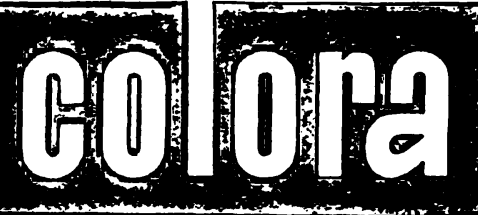




\title{
Klinisches Wörterbuch
}

\author{
Mit klinischen Syndromen
}

von Prof. Dr. med. Dr. phil. W. Pschyrembel '

Gegr. von OtTo DoRnbLÜTH

185.-250., neubearbeitete und erweiterte Auflage

Oktav. Mit 2275 Abbildungen. XVI, 1348 Seiten. 1969.

Ganzleinen DM 28,-

Man wird kaum ein besseres klinisches Wörterbuch als den Pschyrembel finden, das so schnell, zuverlässig und vollständig orientiert, wie dieses altbewährte Nachschlagewerk.

Die Berliner Ärztekammer

In der vorliegenden Auflage des allbekannten „Pschyrembel“ ist auch ein Werk zu begrüßen, das weit über den Rahmen einer Terminologie medizinischer Begriffe hinausgewachsen ist. Neben allen klinischen Fächern umfaßt es nämlich auch wichtigste Begriffe aus der Pharmakologie, der klinischen Chemie, Mikrobiologie, Endokrinologie, Humangenetik, sowie auch die aus dem nuklearmedizinischen Bereich. Es informiert so kurz und so präzise wie möglich auch über die neueste Entwicklung und den Aussagewert klinischer Untersuchungs- und Arbeitsmethoden. Zahlreiche, meistens gelungene photographische Abbildungen und anschauliche Tabellen und Schemata vertiefen das Verständnis einzelner Begriffe.

Parfümerie und Kosmetik

Auch im nuklearmedizinischen Bereich wurde das Fachgebiet der Radiologie vollständig neu bearbeitet und damit den neuesten wissenschaftlichen Erkenntnissen und technischen Neuerungen angepaßt.

Deutsche Hebammen-Zeitschrift

Walter de Gruyter \& Co - Berlin 30 